\title{
Real interpolation of spaces of differential forms
}

\section{Journal Article}

\section{Author(s):}

Hiptmair, Ralf; Li, Jingzhi; Zou, Jun

Publication date:

2012-02

Permanent link:

https://doi.org/10.3929/ethz-b-000158479

Rights / license:

In Copyright - Non-Commercial Use Permitted

Originally published in:

Mathematische Zeitschrift 120(1-2), https://doi.org/10.1007/s00209-010-0803-5 


\title{
Real interpolation of spaces of differential forms
}

\author{
Ralf Hiptmair · Jingzhi Li · Jun Zou
}

Received: 27 July 2009 / Accepted: 26 July 2010 / Published online: 20 November 2010

C Springer-Verlag 2010

\begin{abstract}
In this paper, we study interpolation of Hilbert spaces of differential forms using the real method of interpolation. We show that the scale of fractional order Sobolev spaces of differential $l$-forms in $H^{s}$ with exterior derivative in $H^{s}$ can be obtained by real interpolation. Our proof heavily relies on the recent discovery of smoothed Poincaré lifting for differential forms [M. COSTABEL AND A. MCINTOSH, On Bogovskii and regularized Poincare integral operators for de Rham complexes on Lipschitz domains, Math. Z. 265(2): 297-320, 2010]. They enable the construction of universal extension operators for Sobolev spaces of differential forms, which, in turns, pave the way for a Fourier transform based proof of equivalences of $K$-functionals.
\end{abstract}

Keywords Differential forms · Fractional Sobolev spaces · Real interpolation · K-functional $\cdot$ Smoothed Poincaré lifting $\cdot$ Universal extension

Mathematics Subject Classification (2000) $\quad 46 \mathrm{~B} 70 \cdot 47 \mathrm{~A} 57$

\section{Introduction}

We consider a bounded Lipschitz domain $\Omega \subset \mathbb{R}^{d}, d \in \mathbb{N}$ and $d \geq 2$. Let $\Lambda^{l}$ represent the vector space of real-valued (or complex-valued), alternating, $l$-multilinear maps on $\mathbb{R}^{d}$,

The work of J. Zou was substantially supported by Hong Kong RGC grants (Projects 404606 and 404407).

R. Hiptmair $(\varangle) \cdot$ J. Li

SAM, ETH Zurich, Zurich 8092, Switzerland

e-mail: hiptmair@sam.math.ethz.ch

J. Li

e-mail: jingzhi.li@sam.math.ethz.ch

J. Zou

Department of Mathematics, The Chinese University of Hong Kong, Shatin, N.T., Hong Kong

e-mail: zou@math.cuhk.edu.hk 
which is of dimension $\left(\begin{array}{l}d \\ l\end{array}\right)$. A differential form of order $l$ on $\Omega$ is a mapping $\Omega \mapsto \Lambda^{l}$. Given an increasing $l$-permutation $I=\left(i_{1}, \ldots, i_{l}\right), 1 \leq i_{1}<i_{2}<\cdots<i_{l} \leq d, 1 \leq l \leq d$, we introduce the basis $l$-form $\boldsymbol{d} \mathbf{x}_{I}=\boldsymbol{d} x_{i_{1}} \wedge \cdots \wedge \boldsymbol{d} x_{i_{l}}$, where $\boldsymbol{d} x_{i}$ 's are the canonical coordinate forms in $\mathbb{R}^{d}$. This basis representation permits us to introduce the Hilbert spaces

$$
\boldsymbol{H}^{s}\left(\Omega, \Lambda^{l}\right):=\left\{\omega=\sum_{I} \omega_{I} \boldsymbol{d} \mathbf{x}_{I}: \omega_{I} \in H^{s}(\Omega)\right\}, \quad s \in \mathbb{R}_{0}^{+},
$$

where $H^{s}(\Omega)=W^{s, 2}(\Omega)$ is the standard $L^{2}(\Omega)$-based Sobolev space (of equivalence classes of functions $\Omega \mapsto \mathbb{R}$ ) of fractional order $s$. Throughout, $\Sigma_{I}$ means the summation over all the increasing $l$-permutations $I$ and $\mathbb{R}_{0}^{+}:=\{s \mid s \geq 0\}$. Recall that $\Lambda^{0}$ can be identified with $\mathbb{R}$ and $\boldsymbol{H}^{s}\left(\Omega, \Lambda^{0}\right)$ with $H^{s}(\Omega)$. It is known [10, Thm. B.8] that these fractional spaces form a scale of interpolation spaces, namely

$$
\text { for } 0<\theta<1, \quad s_{0}, s_{1} \in \mathbb{R}, \quad s=(1-\theta) s_{0}+\theta s_{1} \Rightarrow H^{s}(\Omega)=\left[H^{s_{0}}(\Omega), H^{s_{1}}(\Omega)\right]_{\theta},
$$

where $[X, Y]_{\theta}$ designates the space obtained by real interpolation between the Banach spaces $X$ and $Y$, see [13], [2, Ch. 3] and Section 2. As a consequence, the spaces $\boldsymbol{H}^{s}\left(\Omega, \Lambda^{l}\right)$ also form a scale of interpolation spaces.

Writing $\boldsymbol{d}$ for the exterior derivative, the spaces

$$
\boldsymbol{H}^{s}\left(\boldsymbol{d}, \Omega, \Lambda^{l}\right):=\left\{\boldsymbol{\omega} \in \boldsymbol{H}^{s}\left(\boldsymbol{d}, \Omega, \Lambda^{l}\right) \mid \boldsymbol{d} \boldsymbol{\omega} \in \boldsymbol{H}^{s}\left(\boldsymbol{d}, \Omega, \Lambda^{l+1}\right)\right\}, \quad s \in \mathbb{R}_{0}^{+},
$$

play a key role in the statement of second-order variational boundary value problems for differential forms, $c f$. [11]. In this article we give a positive answer to the question, whether these Hilbert spaces $\boldsymbol{H}^{s}\left(\boldsymbol{d}, \Omega, \Lambda^{l}\right), 0 \leq l \leq d$, are related by real interpolation analogous to (1.2). More precisely, in Sect. 4 we will prove the following main result:

Theorem 1 Let $\Omega$ be a bounded Lipschitz domain. For $s_{0}, s_{1} \in \mathbb{R}_{0}^{+}$and $0 \leq l \leq d$,

$$
\left[\boldsymbol{H}^{s_{0}}\left(\boldsymbol{d}, \Omega, \Lambda^{l}\right), \boldsymbol{H}^{s_{1}}\left(\boldsymbol{d}, \Omega, \Lambda^{l}\right)\right]_{\theta}=\boldsymbol{H}^{s}\left(\boldsymbol{d}, \Omega, \Lambda^{l}\right)
$$

with equivalent norms, where $s=(1-\theta) s_{0}+\theta s_{1}$ for $0<\theta<1$.

The policy of the proof of Theorem 1, which is elaborated in Sect. 4, is as follows: first we show the assertion for $\Omega=\mathbb{R}^{d}$ by means of Fourier techniques. Then the problem for general bounded domains is reduced to that case by means of a universal extension theorem for the spaces $\boldsymbol{H}^{s}\left(\boldsymbol{d}, \Omega, \Lambda^{l}\right)$. To that end, we rely on E. Stein's classical extension operator. How this is done employing a smoothed Poincaré mapping is outlined in Sect. 3.

We remind that interpolation in function spaces is a powerful theoretical tool in functional analysis and numerical analysis, because estimates obtained for (simpler) special cases can instantly be extended to a whole scale of spaces. The spaces $\boldsymbol{H}^{s}\left(\boldsymbol{d}, \Omega, \Lambda^{l}\right)$ of differential forms discussed in this paper are isomorphic to the Sobolev spaces $\boldsymbol{H}(\operatorname{div} ; \Omega), \boldsymbol{H}(\mathbf{c u r l} ; \Omega)$ for $d=3$. These Sobolev spaces play a key role in the variational statement and regularity theory for boundary value problems in fluid mechanics and electromagnetics $[8,6,4,5]$. An interpolation theory for theses spaces will have significance for the mathematical and numerical analysis of these boundary value problems.

Despite the evident usefulness of Theorem 1 it seems not to be available in the literature. We mention the abstract framework of [1], but verifying its assumptions for the concrete setting discussed in this paper appears to be challenging. 
Remark 1 To keep the presentation simple, we confine ourselves to the Hilbert space setting of spaces based on $L^{2}(\Omega)$. Extension to $L^{p}(\Omega)$-settings, $1 \leq p \leq \infty$ is likely possible by generalizing our approach.

\section{Real method of interpolation}

Let us first recall the real method of interpolation (cf. [2, Ch. 3], [10, App. B], [3, Ch. 14] for details). Assume a compatible pair of Hilbert spaces $\mathcal{X}_{0}$ and $\mathcal{X}_{1}$ with continuous embedding $\mathcal{X}_{1} \subset \mathcal{X}_{0}$. By the real method of interpolation, we can define for $0<\theta<1$ a family of interpolation spaces $\left[\mathcal{X}_{0}, \mathcal{X}_{1}\right]_{\theta}$ with the following nesting property

$$
\mathcal{X}_{1} \subset\left[\mathcal{X}_{0}, \mathcal{X}_{1}\right]_{\theta} \subset \mathcal{X}_{0}
$$

The $\left[\mathcal{X}_{0}, \mathcal{X}_{1}\right]_{\theta}$-norm is defined through Peetre's $K$-functional by

$$
\begin{aligned}
& \|\mathbf{v}\|_{\left[\mathcal{X}_{0}, \mathcal{X}_{1}\right]_{\theta}}=\left(\int_{0}^{\infty}\left(t^{-\theta} K(t, \mathbf{v})\right)^{2} \frac{\mathrm{d} t}{t}\right)^{1 / 2}, \\
& K(t, \mathbf{v})^{2}:=\inf _{\substack{\mathbf{v}=\mathbf{v}_{0}+\mathbf{v}_{1} \\
\mathbf{v}_{0} \in \mathcal{X}_{0}, \mathbf{v}_{1} \in \mathcal{X}_{1}}}\left\{\left\|\mathbf{v}_{0}\right\|_{\mathcal{X}_{0}}^{2}+t^{2}\left\|\mathbf{v}_{1}\right\|_{\mathcal{X}_{1}}^{2}\right\} .
\end{aligned}
$$

For well-known properties of interpolation spaces and families of linear operators defined on them, the reader is referred to $[2,13]$.

\section{Universal extension}

We start from a celebrated extension theorem for Sobolev spaces due to E. M. Stein, see [12, Theorem 5, pp.181]:

Theorem 2 For a bounded Lipschitz domain $\Omega \subset \mathbb{R}^{d}(d \in \mathbb{N}, d \geq 2)$ there is an operator $\mathscr{E}: C^{\infty}(\bar{\Omega}) \mapsto C^{\infty}\left(\mathbb{R}^{d}\right)$ which satisfies

1. (extension property) $\mathscr{E} u(\mathbf{x})=u(\mathbf{x})$ for all $\mathbf{x} \in \bar{\Omega}$, and

2. (continuity) for any $m \in \mathbb{N}_{0}$ there exists a constant $C=C(m, \Omega)$ such that

$$
\|\mathscr{E} u\|_{H^{m}\left(\mathbb{R}^{d}\right)} \leq C\|u\|_{H^{m}(\Omega)} \quad \forall u \in C^{\infty}(\bar{\Omega}) .
$$

Thus, $\mathscr{E}$ can be extended to a continuous extension operator $\mathscr{E}: H^{m}(\Omega) \mapsto H^{m}\left(\mathbb{R}^{d}\right)$ for any $m \in \mathbb{N}$ by a density argument. Furthermore, in light of of (1.2), by interpolation [10, Theorem B.2] the operator $\mathscr{E}$ can be generalized to fractional Sobolev spaces, that is, $\mathscr{E}: H^{s}(\Omega) \mapsto H^{s}\left(\mathbb{R}^{d}\right)$ is continuous for any $s \in \mathbb{R}_{0}^{+}$. Due to the definition in (1.1), by componentwise application, we obtain an extension operator still denoted by $\mathscr{E}: \boldsymbol{H}^{s}\left(\Omega, \Lambda^{l}\right) \mapsto$ $\boldsymbol{H}^{s}\left(\mathbb{R}^{d}, \Lambda^{l}\right)$ for any $s \in \mathbb{R}_{0}^{+}$and $0 \leq l \leq d$. The operator $\mathscr{E}$ may be called "universal" for its one-formula-fits-all elegance.

A similar operator for the spaces $\boldsymbol{H}^{s}\left(\boldsymbol{d}, \Omega, \Lambda^{l}\right)$ will be a key technical tool in our approach to interpolation spaces. It will be based on some so-called smoothed Poincaré liftings recently introduced by M. Costabel and A. McIntosh in [7], where they used it to prove the following theorem [7, Theorem 4.6]: 
Theorem 3 Let $\Omega \subset \mathbb{R}^{d}$ be a bounded Lipschitz domain, then for $l=0,1, \ldots, d$, there exist pseudodifferential operators $R_{l}$ and $K_{l}$ with the following properties:

1. For any $s \in \mathbb{R}, R_{l}$ maps from $\boldsymbol{H}^{s}\left(\Omega, \Lambda^{l}\right)$ into $\boldsymbol{H}^{s+1}\left(\Omega, \Lambda^{l-1}\right)$ continuously and $K_{l}$ maps from $\boldsymbol{H}^{s}\left(\Omega, \Lambda^{l}\right)$ into $\boldsymbol{H}^{t}\left(\Omega, \Lambda^{l}\right)$ continuously for any $t \in \mathbb{R}$.

2. For any $\boldsymbol{\omega} \in \boldsymbol{H}^{s}\left(\boldsymbol{d}, \Omega, \Lambda^{l}\right)$, there holds the identity

$$
d R_{l} \omega+R_{l+1} d \omega+K_{l} \omega=\omega \text { in } \Omega .
$$

This theorem paves the way for harnessing the classical Stein extension operator $\mathscr{E}$ from Theorem 2 to build universal extension operators $\mathscr{C}_{l}: \boldsymbol{H}^{s}\left(\boldsymbol{d}, \Omega, \Lambda^{l}\right) \mapsto \boldsymbol{H}^{s}\left(\boldsymbol{d}, \mathbb{R}^{d}, \Lambda^{l}\right)$ for $s \in \mathbb{R}_{0}^{+}$, according to

$$
\mathscr{C}_{l}:= \begin{cases}\boldsymbol{d} \circ \mathscr{E} \circ R_{l}+\mathscr{E} \circ R_{l+1} \circ \boldsymbol{d}+\mathscr{E} \circ K_{l}, & l=0,1, \ldots, d-1 ; \\ \boldsymbol{d} \circ \mathscr{E} \circ R_{l}+\mathscr{E} \circ K_{l} . & l=d .\end{cases}
$$

Now we can show a universal extension theorem for the Sobolev spaces of differential forms $\boldsymbol{H}^{s}\left(\boldsymbol{d}, \Omega, \Lambda^{l}\right)$.

Theorem 4 For a bounded Lipschitz domain $\Omega \subset \mathbb{R}^{d}(d \in \mathbb{N}, d \geq 2)$ there is an operator $\mathscr{C}_{l}: \boldsymbol{H}^{s}\left(\boldsymbol{d}, \Omega, \Lambda^{l}\right) \mapsto \boldsymbol{H}^{s}\left(\boldsymbol{d}, \mathbb{R}^{d}, \Lambda^{l}\right), s \in \mathbb{R}_{0}^{+}$, which satisfies

1. (extension property) $\mathscr{C}_{l} \boldsymbol{\omega}(\mathbf{x})=\omega(\mathbf{x})$ a.e. in $\Omega$, and

2. (continuity) for any $0 \leq l \leq d$ there exists a constant $C=C(\Omega, s)$ such that

$$
\|\mathscr{C} \boldsymbol{\omega}\|_{\boldsymbol{H}^{s}\left(\boldsymbol{d}, \mathbb{R}^{d}, \Lambda^{l}\right)} \leq C\|\boldsymbol{\omega}\|_{\boldsymbol{H}^{s}\left(\boldsymbol{d}, \Omega, \Lambda^{l}\right)} \quad \forall \boldsymbol{\omega} \in \boldsymbol{H}^{s}\left(\boldsymbol{d}, \Omega, \Lambda^{l}\right) .
$$

Proof Let $\boldsymbol{\omega} \in \boldsymbol{H}^{s}\left(\boldsymbol{d}, \Omega, \Lambda^{l}\right)$, namely $\boldsymbol{\omega} \in \boldsymbol{H}^{s}\left(\Omega, \Lambda^{l}\right)$ and $\boldsymbol{d} \boldsymbol{\omega} \in \boldsymbol{H}^{s}\left(\Omega, \Lambda^{l+1}\right)$. Note that $l=d$ is a degenerate case, since then $\boldsymbol{d} \boldsymbol{\omega}=0$, and the assertion of the theorem becomes trivial. Hence, we restrict ourselves to $0 \leq l<d$. One possible formula for the extension operator is given in (3.2). Then it is immediate to see that $(\mathscr{C} \omega)_{\left.\right|_{\Omega}}=\boldsymbol{\omega}$ from (3.1) and $\mathscr{C} \boldsymbol{\omega} \in \boldsymbol{H}^{s}\left(\boldsymbol{d}, \mathbb{R}^{d}, \Lambda^{l}\right)$. The continuity holds since

$$
\begin{aligned}
& \|\mathscr{C} \boldsymbol{\omega}\|_{\boldsymbol{H}^{s}\left(\boldsymbol{d}, \mathbb{R}^{d}, \Lambda^{l}\right)} \leq\left\|\boldsymbol{d} \circ \mathscr{E} \circ R_{l} \boldsymbol{\omega}+\mathscr{E} \circ R_{l+1} \circ \boldsymbol{d} \boldsymbol{\omega}\right\|_{\boldsymbol{H}^{s}\left(\mathbb{R}^{d}, \Lambda^{l}\right)} \\
& +\left\|\boldsymbol{d} \circ \mathscr{E} \circ R_{l+1} \circ \boldsymbol{d} \boldsymbol{\omega}\right\|_{\boldsymbol{H}^{s}\left(\mathbb{R}^{d}, \Lambda^{l}\right)}+\left\|\mathscr{E} \circ K_{l} \boldsymbol{\omega}\right\|_{\boldsymbol{H}^{s}\left(\boldsymbol{d}, \mathbb{R}^{d}, \Lambda^{l}\right)} \\
\leq & C\left(\left\|\mathscr{E} \circ R_{l} \boldsymbol{\omega}\right\|_{\boldsymbol{H}^{s+1}\left(\mathbb{R}^{d}, \Lambda^{l-1}\right)}+\left\|\mathscr{E} \circ R_{l+1} \circ \boldsymbol{d} \boldsymbol{\omega}\right\|_{\boldsymbol{H}^{s+1}\left(\mathbb{R}^{d}, \Lambda^{l}\right)}+\left\|\mathscr{E} \circ K_{l} \boldsymbol{\omega}\right\|_{\boldsymbol{H}^{s+1}\left(\mathbb{R}^{d}, \Lambda^{l}\right)}\right) \\
\leq & C\left(\left\|R_{l} \boldsymbol{\omega}\right\|_{\boldsymbol{H}^{s+1}\left(\Omega, \Lambda^{l-1}\right)}+\left\|R_{l+1} \circ \boldsymbol{d} \boldsymbol{\omega}\right\|_{\boldsymbol{H}^{s+1}\left(\Omega, \Lambda^{l}\right)}+\left\|K_{l} \boldsymbol{\omega}\right\|_{\boldsymbol{H}^{s+1}\left(\Omega, \Lambda^{l}\right)}\right) \\
\leq & C\left(\|\boldsymbol{\omega}\|_{\boldsymbol{H}^{s}\left(\Omega, \Lambda^{l}\right)}+\|\boldsymbol{d} \boldsymbol{\omega}\|_{\boldsymbol{H}^{s}\left(\Omega, \Lambda^{l+1}\right)}\right) \leq C\|\boldsymbol{\omega}\|_{\boldsymbol{H}^{s}\left(\boldsymbol{d}, \Omega, \Lambda^{l}\right)} .
\end{aligned}
$$

where we use the triangle inequality first and employ the continuity of the Stein operator $\mathscr{E}$ and Theorem 3 in the third and fourth inequalities, respectively. This completes the proof.

\section{Interpolation in $H^{s}\left(d, \Omega, \Lambda^{l}\right)$}

In this section, we establish the equivalence between the interpolation spaces and fractional order Sobolev spaces $\boldsymbol{H}^{s}\left(\boldsymbol{d}, \Omega, \Lambda^{l}\right)$ of differential forms.

In the first step, we establish the interpolation theorem about the equivalence between fractional Sobolev spaces $\boldsymbol{H}^{s}\left(\boldsymbol{d}, \mathbb{R}^{d}, \Lambda^{l}\right)$ and interpolation spaces for the domain $\mathbb{R}^{d}$. For 
$0<\theta<1, s_{0}, s_{1} \in \mathbb{R}$ with $s_{0}<s_{1}$, and $s=(1-\theta) s_{0}+\theta s_{1}$, let us recall the definition of the $\left[\boldsymbol{H}^{s_{0}}\left(\boldsymbol{d}, \mathbb{R}^{d}, \Lambda^{l}\right), \boldsymbol{H}^{s_{1}}\left(\boldsymbol{d}, \mathbb{R}^{d}, \Lambda^{l}\right)\right]_{\theta}$-norm of the interpolation space via the K-functional:

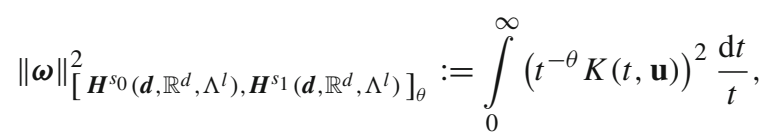

where

$$
K(t, \boldsymbol{\omega})^{2}:=\inf _{\substack{\boldsymbol{\omega}=\boldsymbol{\omega}_{0}+\omega_{1} \\ \boldsymbol{\omega}_{0} \in \boldsymbol{H}^{s_{0}}\left(\boldsymbol{d}, \mathbb{R}^{d}, \Lambda^{l}\right) \\ \omega_{1} \in \boldsymbol{H}^{s_{1}}\left(\boldsymbol{d}, \mathbb{R}^{d}, \Lambda^{l}\right)}}\left\{\|\boldsymbol{\omega}\|_{\boldsymbol{H}^{s_{0}}\left(\boldsymbol{d}, \mathbb{R}^{d}, \Lambda^{l}\right)}^{2}+t^{2}\|\boldsymbol{\omega}\|_{\boldsymbol{H}^{s_{1}}\left(\boldsymbol{d}, \mathbb{R}^{d}, \Lambda^{l}\right)}^{2}\right\}
$$

On the other hand, for any $\boldsymbol{\omega} \in \boldsymbol{H}^{s}\left(\boldsymbol{d}, \mathbb{R}^{d}, \Lambda^{l}\right)$, the $\boldsymbol{H}^{s}\left(\boldsymbol{d}, \mathbb{R}^{d}, \Lambda^{l}\right)$-norm of the fractional order Sobolev spaces is defined by

$$
\|\boldsymbol{\omega}\|_{\boldsymbol{H}^{s}\left(\mathbb{R}^{d}, \Lambda^{l}\right)}^{2}:=\int_{\mathbb{R}^{d}}\left(1+|\boldsymbol{\xi}|^{2}\right)^{s}|\widehat{\boldsymbol{\omega}}(\boldsymbol{\xi})|^{2} \mathrm{~d} \boldsymbol{\xi}
$$

where $\widehat{\omega}$ is the Fourier transform of $\omega$, and $|\widehat{\omega}(\xi)|^{2}:=\sum_{I}\left|\widehat{\omega}_{I}(\xi)\right|^{2}$. Here the Fourier transform of a differential $l$-form $\boldsymbol{\omega}=\sum_{I} \boldsymbol{\omega}_{I} \boldsymbol{d} \mathbf{x}_{I} \in \boldsymbol{L}^{2}\left(\mathbb{R}^{d} ; \Lambda^{l}\right)$, still denoted by $\mathscr{F}$, is defined componentwise by

$$
\widehat{\omega}(\xi):=\mathscr{F}(\omega)(\xi)=\sum_{I} \widehat{\omega}_{I}(\xi) d \xi_{I}
$$

where

$$
\widehat{\boldsymbol{\omega}}_{I}(\boldsymbol{\xi}):=\mathscr{F}\left(\boldsymbol{\omega}_{I}\right)(\boldsymbol{\xi})=\frac{1}{(2 \pi)^{d / 2}} \int_{\mathbb{R}^{d}} \exp (-\imath \boldsymbol{\xi} \cdot \mathbf{x}) \boldsymbol{\omega}_{I}(\mathbf{x}) \mathrm{d} \mathbf{x},
$$

and $\iota$ is the imaginary unit, $\xi=\left(\xi_{1}, \cdots, \xi_{d}\right)^{T}$ is the vectorial angular frequency in $\mathbb{R}^{d}$ and $\boldsymbol{d} \boldsymbol{\xi}_{I}=\boldsymbol{d} \xi_{i_{1}} \wedge \cdots \wedge \boldsymbol{d} \xi_{i_{l}}$, with $I$ being an increasing $l$-permutation. Note that (4.3) corresponds to a componentwise definition of the norm for Sobolev spaces of differential forms by means of the Fourier transform method (cf. [10, Ch. 3]).

It is easy to see that the Fourier transform converts the exterior derivative into an exterior product:

Lemma 1 For any $\boldsymbol{\omega} \in \boldsymbol{H}\left(\boldsymbol{d}, \Omega, \Lambda^{l}\right)$, we have

$$
\mathscr{F}(\boldsymbol{d} \omega)=\imath \widehat{\xi} \wedge \mathscr{F}(\boldsymbol{\omega}),
$$

where $\widehat{\boldsymbol{\xi}}$ is the differential 1 -form in the frequency domain, namely $\widehat{\boldsymbol{\xi}}:=\xi_{1} \boldsymbol{d} \xi_{1}+\xi_{2} \boldsymbol{d} \xi_{2}+$ $\cdots+\xi_{d} d \xi_{d}$.

Thus by Lemma 1, we may write

$$
\begin{aligned}
\|\boldsymbol{\omega}\|_{\boldsymbol{H}^{s}\left(\boldsymbol{d}, \mathbb{R}^{d}, \Lambda^{l}\right)}^{2} & =\|\boldsymbol{\omega}\|_{\boldsymbol{H}^{s}\left(\mathbb{R}^{d}, \Lambda^{l}\right)}^{2}+\|\boldsymbol{d} \boldsymbol{\omega}\|_{\boldsymbol{H}^{s}\left(\mathbb{R}^{d}, \Lambda^{l}\right)}^{2} \\
& =\int_{\mathbb{R}^{d}}\left(1+|\boldsymbol{\xi}|^{2}\right)^{s}\left(|\widehat{\boldsymbol{\omega}}(\boldsymbol{\xi})|^{2}+\left.\widehat{\boldsymbol{\xi}} \wedge \widehat{\boldsymbol{\omega}}(\boldsymbol{\xi})\right|^{2}\right) \mathrm{d} \boldsymbol{\xi} .
\end{aligned}
$$

To show the equivalence, we need a technical lemma which generalizes [10, Ex. B.4] to space $\Lambda^{l}$ of complex-valued alternating, $l$-multilinear mappings. 
Lemma 2 For any two constants $c_{0}>0$ and $c_{1}>0$, and some $z \in \Lambda^{l}$, it holds that

$$
\min _{\substack{z=z_{0}+z_{1} \\ z_{0}, z_{1} \in \Lambda^{l}}}\left(c_{0}\left|z_{0}\right|^{2}+c_{1}\left|z_{1}\right|^{2}\right)=\frac{c_{0} c_{1}}{c_{0}+c_{1}}|z|^{2},
$$

and the minimum is achieved when $c_{0} z_{0}=c_{1} z_{1}=c_{0} c_{1} z /\left(c_{0}+c_{1}\right)$.

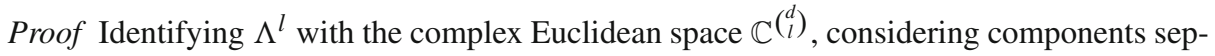
arately and splitting into real and imaginary parts, (4.5) is seen to be equivalent to

$$
\min _{\substack{x=x_{0}+x_{1} \\ x_{0}, x_{1} \in \mathbb{R}}}\left(c_{0} x_{0}^{2}+c_{1} x_{1}^{2}\right)=\frac{c_{0} c_{1}}{c_{0}+c_{1}} x^{2}, \quad x \in \mathbb{R} .
$$

Then the proof boils down to an exercise in the use of Lagrangian multipliers.

Now we can establish the equivalence of the fractional Sobolev spaces of differential forms $\boldsymbol{H}^{s}\left(\boldsymbol{d}, \mathbb{R}^{d}, \Lambda^{l}\right)$ and the interpolation spaces $\left[\boldsymbol{H}^{s_{0}}\left(\boldsymbol{d}, \mathbb{R}^{d}, \Lambda^{l}\right), \boldsymbol{H}^{s_{1}}\left(\boldsymbol{d}, \mathbb{R}^{d}, \Lambda^{l}\right)\right]_{s}$.

Lemma 3 For $s_{0}, s_{1} \in \mathbb{R}$ with $s_{0}<s_{1}$, and $l \in \mathbb{N}_{0}$ with $0 \leq l \leq d$, it holds that

$$
\left[\boldsymbol{H}^{s_{0}}\left(\boldsymbol{d}, \mathbb{R}^{d}, \Lambda^{l}\right), \boldsymbol{H}^{s_{1}}\left(\boldsymbol{d}, \mathbb{R}^{d}, \Lambda^{l}\right)\right]_{\theta}=\boldsymbol{H}^{s}\left(\boldsymbol{d}, \mathbb{R}^{d}, \Lambda^{l}\right),
$$

with equivalent norms, where $s=(1-\theta) s_{0}+\theta s_{1}$ for $0<\theta<1$.

Proof We take the cue from the proof of the interpolation theorem for standard Sobolev spaces on $\mathbb{R}^{d}\left[10\right.$, Thm B.7]. For any $\boldsymbol{\omega} \in \boldsymbol{H}^{s}\left(\boldsymbol{d}, \mathbb{R}^{d}, \Lambda^{l}\right)$, let $\boldsymbol{\omega}=\boldsymbol{\omega}_{0}+\boldsymbol{\omega}_{1}$ with $\boldsymbol{\omega}_{j} \in \boldsymbol{H}^{s_{j}}\left(\boldsymbol{d}, \mathbb{R}^{d}, \Lambda^{l}\right)$ for $j=0,1$. We observe that

$$
\begin{aligned}
& K(t, \boldsymbol{\omega})^{2}=\inf _{\substack{\boldsymbol{\omega}=\boldsymbol{\omega}_{0}+\boldsymbol{\omega}_{1} \\
\boldsymbol{\omega}_{0} \in \boldsymbol{H}^{s_{0}}\left(\boldsymbol{d}, \mathbb{R}^{d}, \Lambda^{l}\right) \\
\boldsymbol{\omega}_{1} \in \boldsymbol{H}^{s_{1}}\left(\boldsymbol{d}, \mathbb{R}^{d}, \Lambda^{l}\right)}}\left\{\left\|\boldsymbol{\omega}_{0}\right\|_{\boldsymbol{H}^{s_{0}}\left(\boldsymbol{d}, \mathbb{R}^{d}, \Lambda^{l}\right)}^{2}+t^{2}\left\|\boldsymbol{\omega}_{1}\right\|_{\left.\boldsymbol{H}^{s_{1}}\left(\boldsymbol{d}, \mathbb{R}^{d}, \Lambda^{l}\right)\right\}}^{2}\right\} \\
= & \inf _{\widehat{\boldsymbol{\omega}}=\widehat{\boldsymbol{\omega}}_{0}+\widehat{\boldsymbol{\omega}}_{1}} \int_{\mathbb{R}^{d}}\left[\left(1+|\boldsymbol{\xi}|^{2}\right)^{s_{0}}\left(\left|\widehat{\boldsymbol{\omega}}_{0}(\boldsymbol{\xi})\right|^{2}+\left|\widehat{\boldsymbol{\xi}} \wedge \widehat{\boldsymbol{\omega}}_{0}(\boldsymbol{\xi})\right|^{2}\right)\right. \\
& \left.+t^{2}\left(1+|\boldsymbol{\xi}|^{2}\right)^{s_{1}}\left(\left|\widehat{\boldsymbol{\omega}}_{1}(\boldsymbol{\xi})\right|^{2}+\left|\widehat{\boldsymbol{\xi}} \wedge \widehat{\boldsymbol{\omega}}_{1}(\boldsymbol{\xi})\right|^{2}\right)\right] \mathrm{d} \boldsymbol{\xi} \\
\geq & \inf _{\widehat{\boldsymbol{\omega}}=\widehat{\boldsymbol{\omega}}_{0}+\widehat{\boldsymbol{\omega}}_{1}} \int_{\mathbb{R}^{d}}\left[\left(1+|\boldsymbol{\xi}|^{2}\right)^{s_{0}}\left(\left|\widehat{\boldsymbol{\omega}}_{0}(\boldsymbol{\xi})\right|^{2}\right)+t^{2}\left(1+|\boldsymbol{\xi}|^{2}\right)^{s_{1}}\left(\left|\widehat{\boldsymbol{\omega}}_{1}(\boldsymbol{\xi})\right|^{2}\right)\right] \mathrm{d} \boldsymbol{\xi} \\
& +\inf _{\widehat{\boldsymbol{\omega}}=\widehat{\boldsymbol{\omega}}_{0}+\widehat{\boldsymbol{\omega}}_{1}} \int\left[\left(1+|\boldsymbol{\xi}|^{2}\right)^{s_{0}}\left(\left|\widehat{\boldsymbol{\xi}} \wedge \widehat{\boldsymbol{\omega}}_{0}(\boldsymbol{\xi})\right|^{2}\right)+t^{2}\left(1+|\boldsymbol{\xi}|^{2}\right)^{s_{1}}\left(\left|\widehat{\boldsymbol{\xi}} \wedge \widehat{\boldsymbol{\omega}}_{1}(\boldsymbol{\xi})\right|^{2}\right)\right] \mathrm{d} \boldsymbol{\xi}=: \mathfrak{S}+\mathfrak{T}
\end{aligned}
$$

where $\widehat{\boldsymbol{\omega}}_{j}(\boldsymbol{\xi})$ is the Fourier transform of $\boldsymbol{\omega}_{j}$ for $j=0,1$ and $\widehat{\boldsymbol{\omega}}(\boldsymbol{\xi})=\widehat{\boldsymbol{\omega}}_{0}(\boldsymbol{\xi})+\widehat{\boldsymbol{\omega}}_{1}(\boldsymbol{\xi})$ by the linearity of the Fourier transform. By Lemma 2, we see that for each $\boldsymbol{\xi}$ the integrand in $\mathfrak{S}$ is minimized when

$$
\left(1+|\boldsymbol{\xi}|^{2}\right)^{s_{0}} \widehat{\boldsymbol{\omega}}_{0}(\boldsymbol{\xi})=t^{2}\left(1+|\boldsymbol{\xi}|^{2}\right)^{s_{1}} \widehat{\boldsymbol{\omega}}_{1}(\boldsymbol{\xi})=\frac{t^{2}\left(1+|\boldsymbol{\xi}|^{2}\right)^{s_{0}+s_{1}}}{\left(1+|\boldsymbol{\xi}|^{2}\right)^{s_{0}}+t^{2}\left(1+|\boldsymbol{\xi}|^{2}\right)^{s_{1}}} \widehat{\boldsymbol{\omega}}(\boldsymbol{\xi}) .
$$

Likewise, by linearity of the operator $\boldsymbol{\xi} \wedge \cdot$, the integrand in $\mathfrak{T}$ is minimized when

$$
\left(1+|\boldsymbol{\xi}|^{2}\right)^{s_{0}} \widehat{\boldsymbol{\xi}} \wedge \widehat{\boldsymbol{\omega}}_{0}(\boldsymbol{\xi})=t^{2}\left(1+|\boldsymbol{\xi}|^{2}\right)^{s_{1}} \widehat{\boldsymbol{\xi}} \wedge \widehat{\boldsymbol{\omega}}_{1}(\boldsymbol{\xi})=\frac{t^{2}\left(1+|\boldsymbol{\xi}|^{2}\right)^{s_{0}+s_{1}}}{\left(1+|\boldsymbol{\xi}|^{2}\right)^{s_{0}}+t^{2}\left(1+|\boldsymbol{\xi}|^{2}\right)^{s_{1}}} \widehat{\boldsymbol{\xi}} \wedge \widehat{\boldsymbol{\omega}}(\boldsymbol{\xi})
$$


Thanks to the special choice of splitting as given in (4.8), (4.9), we have

$$
\begin{aligned}
& K(t, \boldsymbol{\omega})^{2} \geq \int_{\mathbb{R}^{d}} \frac{t^{2}\left(1+|\boldsymbol{\xi}|^{2}\right)^{s_{0}+s_{1}}}{\left(1+|\boldsymbol{\xi}|^{2}\right)^{s_{0}}+t^{2}\left(1+|\boldsymbol{\xi}|^{2}\right)^{s_{1}}}|\widehat{\boldsymbol{\omega}}(\boldsymbol{\xi})|^{2} \mathrm{~d} \boldsymbol{\xi} \\
& +\int_{\mathbb{R}^{d}} \frac{t^{2}\left(1+|\boldsymbol{\xi}|^{2}\right)^{s_{0}+s_{1}}}{\left(1+|\boldsymbol{\xi}|^{2}\right)^{s_{0}}+t^{2}\left(1+|\boldsymbol{\xi}|^{2}\right)^{s_{1}}}|\boldsymbol{\xi} \wedge \widehat{\boldsymbol{\omega}}(\boldsymbol{\xi})|^{2} \mathrm{~d} \boldsymbol{\xi} \\
& \geq \inf _{\widehat{\boldsymbol{\omega}}=\widehat{\boldsymbol{\omega}}_{0}+\widehat{\boldsymbol{\omega}}_{1}} \int_{\mathbb{R}^{d}}\left[\left(1+|\boldsymbol{\xi}|^{2}\right)^{s_{0}}\left(\left|\widehat{\boldsymbol{\omega}}_{0}(\boldsymbol{\xi})\right|^{2}+\left|\widehat{\boldsymbol{\xi}} \wedge \widehat{\boldsymbol{\omega}}_{0}(\boldsymbol{\xi})\right|^{2}\right)\right. \\
& \left.+t^{2}\left(1+|\boldsymbol{\xi}|^{2}\right)^{s_{1}}\left(\left|\widehat{\boldsymbol{\omega}}_{1}(\boldsymbol{\xi})\right|^{2}+\left|\widehat{\boldsymbol{\xi}} \wedge \widehat{\boldsymbol{\omega}}_{1}(\boldsymbol{\xi})\right|^{2}\right)\right] \\
& =K(t, \boldsymbol{\omega})^{2} .
\end{aligned}
$$

Hence we see that, when (4.8), (4.9) hold,

$$
\begin{aligned}
K(t, \boldsymbol{\omega})^{2} & =\int_{\mathbb{R}^{d}} \frac{t^{2}\left(1+|\boldsymbol{\xi}|^{2}\right)^{s_{0}+s_{1}}}{\left(1+|\xi|^{2}\right)^{s_{0}}+t^{2}\left(1+|\boldsymbol{\xi}|^{2}\right)^{s_{1}}}\left(|\widehat{\boldsymbol{\omega}}(\boldsymbol{\xi})|^{2}+|\widehat{\boldsymbol{\xi}} \wedge \widehat{\boldsymbol{\omega}}(\boldsymbol{\xi})|^{2}\right) \mathrm{d} \boldsymbol{\xi} \\
& =\int_{\mathbb{R}^{d}}\left(1+|\boldsymbol{\xi}|^{2}\right)^{s_{0}} f(a(\boldsymbol{\xi}) t)^{2}\left(|\widehat{\boldsymbol{\omega}}(\boldsymbol{\xi})|^{2}+|\widehat{\boldsymbol{\xi}} \wedge \widehat{\boldsymbol{\omega}}(\boldsymbol{\xi})|^{2}\right) \mathrm{d} \boldsymbol{\xi},
\end{aligned}
$$

where $a(\boldsymbol{\xi})=\left(1+|\boldsymbol{\xi}|^{2}\right)^{\left(s_{1}-s_{0}\right) / 2}$ and $f(t)=\frac{t}{\sqrt{1+t^{2}}}$. Therefore we derive for $0<\theta<1$,

$$
\begin{aligned}
& \|\boldsymbol{\omega}\|_{\left[\boldsymbol{H}^{s_{0}}\left(\boldsymbol{d}, \mathbb{R}^{d}, \Lambda^{l}\right), \boldsymbol{H}^{s_{1}}\left(\boldsymbol{d}, \mathbb{R}^{d}, \Lambda^{l}\right)\right]_{\theta}}^{2} \\
& =\int_{\mathbb{R}^{d}}\left(1+|\boldsymbol{\xi}|^{2}\right)^{s_{0}} a(\boldsymbol{\xi})^{2 \theta}\left(\int_{0}^{\infty} \frac{t^{1-2 \theta}}{1+t^{2}} \mathrm{~d} t\right)\left(|\widehat{\boldsymbol{\omega}}(\boldsymbol{\xi})|^{2}+|\widehat{\boldsymbol{\xi}} \wedge \widehat{\boldsymbol{\omega}}(\boldsymbol{\xi})|^{2}\right) \mathrm{d} \boldsymbol{\xi} \\
& =\frac{\pi}{2 \sin \pi \theta} \int_{\mathbb{R}^{d}}\left(1+|\boldsymbol{\xi}|^{2}\right)^{s}\left(|\widehat{\boldsymbol{\omega}}(\boldsymbol{\xi})|^{2}+|\widehat{\boldsymbol{\xi}} \wedge \widehat{\boldsymbol{\omega}}(\boldsymbol{\xi})|^{2}\right) \mathrm{d} \boldsymbol{\xi}=\frac{\pi}{2 \sin \pi \theta}\|\boldsymbol{\omega}\|_{\boldsymbol{H}^{s}\left(\boldsymbol{d}, \mathbb{R}^{d}, \Lambda^{l}\right)}^{2} .
\end{aligned}
$$

This completes the proof.

Now we are in a position to prove our main result Theorem 1 about the equivalence of interpolation spaces in bounded Lipschitz domains.

proof of Theorem 1 We introduce the canonical restriction operators

$$
T_{l}: \boldsymbol{H}^{s}\left(\boldsymbol{d}, \mathbb{R}^{d}, \Lambda^{l}\right) \rightarrow \boldsymbol{H}^{s}\left(\boldsymbol{d}, \Omega, \Lambda^{l}\right), \quad T_{l} \omega=\omega_{\mid \Omega},
$$

which restricts differential forms to the domain $\Omega$ and is a contraction for any $s \in \mathbb{R}_{0}^{+}$.

Recalling the extension operator $\mathscr{C}_{l}$ from (3.2), it suffices to show that the operator product $T_{l} \circ \mathscr{C}_{l}$ defines an isomorphism between $\boldsymbol{H}^{s}\left(\boldsymbol{d}, \Omega, \Lambda^{l}\right)$ and $\left[\boldsymbol{H}^{s_{0}}\left(\boldsymbol{d}, \Omega, \Lambda^{l}\right)\right.$, $\left.\boldsymbol{H}^{s_{1}}\left(\boldsymbol{d}, \Omega, \Lambda^{l}\right)\right]_{\theta}$. To that end we rely on the interpolation theorem (IT) for bounded linear operators [10, Thm. B2] and use that $T_{l} \circ \mathscr{C}_{l}=I d$ universally.

To begin with for $\boldsymbol{\omega} \in \boldsymbol{H}^{s}\left(\boldsymbol{d}, \Omega, \Lambda^{l}\right)$ we find

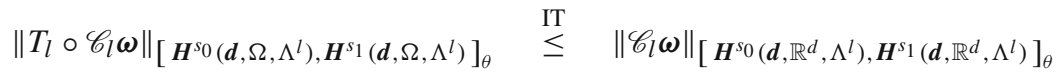

$$
\begin{aligned}
& \stackrel{\text { Lemma }}{\leq} C\left\|\mathscr{C}_{l} \boldsymbol{\omega}\right\|_{\boldsymbol{H}^{s}\left(\boldsymbol{d}, \mathbb{R}^{d}, \Lambda^{l}\right)} \leq C\|\boldsymbol{\omega}\|_{\boldsymbol{H}^{s}\left(\boldsymbol{d}, \Omega, \Lambda^{l}\right)},
\end{aligned}
$$


where the first inequality follows from interpolating the contractions $T_{l}$, the second from Lemma 3, and the third simply from the continuity of $\mathscr{C}_{l}$. Thus, we have established $\boldsymbol{\omega}=\left(T_{l} \circ \mathscr{C}_{l}\right) \boldsymbol{\omega} \in\left[\boldsymbol{H}^{s_{0}}\left(\boldsymbol{d}, \Omega, \Lambda^{l}\right), \boldsymbol{H}^{s_{1}}\left(\boldsymbol{d}, \Omega, \Lambda^{l}\right)\right]_{\theta}$.

Next, for the reverse inclusion, pick any $\boldsymbol{\omega} \in\left[\boldsymbol{H}^{s_{0}}\left(\boldsymbol{d}, \Omega, \Lambda^{l}\right), \boldsymbol{H}^{s_{1}}\left(\boldsymbol{d}, \Omega, \Lambda^{l}\right)\right]_{\theta}$. As above we deduce that

$$
\begin{aligned}
\left\|T_{l} \circ \mathscr{C}_{l} \boldsymbol{\omega}\right\|_{\boldsymbol{H}^{s}\left(\boldsymbol{d}, \Omega, \Lambda^{l}\right)} & \leq\left\|\mathscr{C}_{l} \boldsymbol{\omega}\right\|_{\boldsymbol{H}^{s}\left(\boldsymbol{d}, \mathbb{R}^{d}, \Lambda^{l}\right)} \stackrel{\text { Lemma } 3}{\leq} C\left\|\mathscr{C}_{l} \boldsymbol{\omega}\right\|_{\left[\boldsymbol{H}^{s_{0}}\left(\boldsymbol{d}, \mathbb{R}^{d}, \Lambda^{l}\right), \boldsymbol{H}^{s_{1}}\left(\boldsymbol{d}, \mathbb{R}^{d}, \Lambda^{l}\right)\right]_{\theta}} \\
& \leq C\|\boldsymbol{I T}\|_{\left[\boldsymbol{H}^{s_{0}}\left(\boldsymbol{d}, \Omega, \Lambda^{l}\right), \boldsymbol{H}^{s_{1}}\left(\boldsymbol{d}, \Omega, \Lambda^{l}\right)\right]_{\theta}} \cdot
\end{aligned}
$$

which shows $\boldsymbol{\omega}=\left(T_{l} \circ \mathscr{C}_{l}\right) \boldsymbol{\omega} \in \boldsymbol{H}^{s}\left(\boldsymbol{d}, \Omega, \Lambda^{l}\right)$. This completes the proof.

Remark 2 In three-dimensional Euclidean space $\mathbb{R}^{3}$, we can interpret Theorem 1 for the vector fields modeling differential forms, see, e.g.,[9, Table 2.1]. In particular for the cases $l=1,2$, special cases of the theorem can be stated as follows:

Lemma 4 For $k, m \in \mathbb{N}$ with $k<m$, the following spaces agree

$$
\begin{gathered}
{\left[\boldsymbol{H}^{k}(\operatorname{curl} ; \Omega), \boldsymbol{H}^{m}(\mathbf{c u r l} ; \Omega)\right]_{\theta}=\boldsymbol{H}^{s}(\operatorname{curl} ; \Omega),} \\
{\left[\boldsymbol{H}^{k}(\operatorname{div} ; \Omega), \boldsymbol{H}^{m}(\operatorname{div} ; \Omega)\right]_{\theta}=\boldsymbol{H}^{s}(\operatorname{div} ; \Omega),}
\end{gathered}
$$

with equivalent norms, where $s=(1-\theta) k+\theta m$ for $0<\theta<1$.

Acknowledgment The authors thank the anonymous referee for his careful reading of our manuscript and insightful comments which helped us improve the presentation significantly.

\section{References}

1. Baiocchi, C.: Un teorema di interpolazione; applicazioni ai problemi ai limiti per le equazioni differenziali a derivate parziali. Annali di Matematica Pura Ed Applicata 73(1), 233-251 (1966)

2. Bergh, J., Löfström, J.: Interpolation Spaces. Springer, Berlin (1976)

3. Brenner, S., Scott, R.: Mathematical theory of finite element methods, Texts in Applied Mathematics. 2nd edn. Springer, New York (2002)

4. Costabel, M., Dauge, M.: Singularities of electromagnetic fields in polyhedral domains. Arch. Rational Mech. Anal. 151, 221-276 (2000)

5. Costabel, M., Dauge, M., Nicaise, S.: Singularities of eddy current problems. ESAIM: Math. Model. Numer. Anal. 37(5), 807-831 (2003)

6. Cessenat, M.: Mathematical Methods in Electromagnetism, volume 41 of Advances in Mathematics for Applied Sciences. World Scientific, Singapore (1996)

7. Costabel M., McIntosh A. L: On Bogovskii and regularized Poincare integral operators for de Rham complexes on Lipschitz domains. Math. Z. 265(2), 297-320 (2010)

8. Girault, V., Raviart, P.: Finite element methods for Navier-Stokes equations. Springer, Berlin (1986)

9. Hiptmair, R.: Finite elements in computational electromagnetism. Acta Numerica 11, 237-339 (2002)

10. McLean, W.: Strongly Elliptic Systems and Boundary Integral Equations. Cambridge University Press, Cambridge (2000)

11. Picard, R.: An elementary proof for a compact imbedding result in generalized electromagnetic theory. Math. Z. 187, 151-161 (1984)

12. Stein, E.M.: Singular integrals and differentiability properties of functions. Princeton University Press, Princeton (1970)

13. Triebel, H.: Interpolation theory, function spaces, differential operators. North-Holland, Oxford (1978) 